\title{
ON EXTENDED STRUCTURES OF A CLOSED OPERATOR RELATED TO SEMIGROUP THEORY AND THE ABSTRACT CAUCHY PROBLEM
}

\author{
BY KAI-TAK WONG
}

Communicated by François Treves, July 27, 1973

1. After the fundamental treatise on the semigroup theory by Hille and Phillips, several extensions of the semigroup theory and the abstract Cauchy problem have been developed. Three of the basic developments which are related to the results in this announcement are the distributional semigroups (V. Barbu [1], J. Chazarain [4], I. Ciorănescu [5], G. Da Prato and U. Mosco [6], H. O. Fattorini [9], C. Foiaş [10], D. Fujiwara [11], E. Larsson [16], J. L. Lions [17], J. Peetre [19], L. Schwartz [21], Ushijima [23], K. Yoshinaga [24]), the linear differential equations in Banach space (S. G. Kreln [15] contains a bibliography up to 1966, recently by R. Beals [2], [3], G. Da Prato and Giusti [7], M. Sova [22]), and the semigroup theory on a locally convex space (H. Komatsu [13], Kômura [14], I. Miyadera [18], K. Yosida [25]).

In this communication, we restrict ourselves to consider only a single closed operator $A$ on a Banach space $H$ in order to simplify the statements of our theorems. The problem is the abstract Cauchy problem (ACP), i.e., to find a solution in $H$ for the differential equation $y^{\prime}(t)=A y(t)$ on the interval $[0, \infty)$ with $y(0)=x$ for some $x \in H$. This problem by various authors (for example, Hille and Phillips [12], Kreln [15]) is to find a strongly continuous semigroup $T(t)$ on $[0, \infty)$ of continuous operators on $H$ such that $T(t) x$ is the solution of the problem for certain $x \in H$. In 1960, J. Lions proposed the study of the distributional semigroup which gives a distributional solution for the problem $y^{\prime}(t)-A y(t)=f(t)$. This extends the solvability of the ACP to a larger class of closed operators. What we are trying to do here is to give a formulation in between the two mentioned above. Formally, the idea is similar to that from the theory of partial differential equations, in the sense that the differential operator is solvable in an extended space which is the completion of the underlying space of the differential operator under a weaker topology.

AMS (MOS) subject classifications (1970). Primary 34G05, 47Axx, 47D05; Secondary $35 \mathrm{Kxx}, 35 \mathrm{Lxx}$.

Key words and phrases. Closed operators, abstract Cauchy problem, semigroup of linear transformations. 
An indication of the proof for each theorem stated will be given, and most of the proofs can be obtained by applying the three basic principles in Dunford and Schwarz [8].

2. We will assume throughout this paper that $A$ is a closed operator on a Banach space $H$ with domain $D(A)$ dense in $H$, and the resolvent set $\rho(A) \neq \varnothing$. As usual $H^{*}$ and $A^{*}$ will be the adjoint of $H$ and $A$ respectively. Let us use the notation $D_{H}\left(A^{n}\right)_{s}$ to denote the space $D_{H}\left(A^{n}\right)$ with the Banach norm $\sum_{k=0}^{\infty}\left\|A^{k} x\right\|$. It follows that every element $x$ in $H$ can be naturally identified as an element in $D\left(A^{*}\right)_{s}^{*}$. Let $H_{e}$ be the closure of $H$ in $D\left(A^{*}\right)_{s}^{*}$. Then $A$ as a linear transformation from $D(A)$ to $H_{e}$ can be extended as a continuous linear transformation $A_{e}$ on $H$. Furthermore, $A_{e}$ is a closed operator on the Banach space $H_{e}$ with domain $D_{H_{e}}\left(A_{e}\right)=H$ dense in $H_{e}$, and the resolvent $\rho_{H_{e}}\left(A_{e}\right) \neq \varnothing$. Define $A_{1}=A_{e}$ and $H_{1}=H_{e}$. Inductively, we can define $A_{n+1}=\left(A_{n}\right)_{e}$ and $H_{n+1}=\left(H_{n}\right)_{e}$. On the other hand, denote $A_{r}$ to be the restriction of $A$ on $D\left(A^{2}\right)$. Then $A_{r}$ is a closed operator on $H_{r}=D(A)_{s}$ with domain $D_{H_{r}}\left(A_{r}\right)=D\left(A^{2}\right)$ dense in $H_{r}$, and $\rho_{H_{r}}\left(A_{r}\right)=\varnothing$. Define $H_{-1}=H_{r}$ and $A_{-1}=A_{r}$. Inductively, define for each negative integer $n, H_{n-1}=\left(H_{n}\right)_{r}$ and $A_{n-1}=\left(A_{n}\right)_{r}$. We summarize the properties of $H_{n}$ and $A_{n}$ in the following.

THEOREM 1. There exists a sequence of Banach spaces $H_{n}$ and a sequence of linear transformations $A_{r}$ for each integer $n$ such that

(i) $H_{0}=H$ and $A_{0}=A$;

(ii) $H_{n}$ is contained and dense in $H_{n+1}$;

(iii) $A_{n+1}$ is a closed operator on $H_{n+1}$ with domain $D_{H_{n+1}}\left(A_{n+1}\right)=H_{n}$;

(iv) $H_{n+1}=\left(H_{n}\right)_{e}$ and $A_{n+1}=\left(A_{n}\right)_{e}$;

(v) conditions (i) to (iv) characterize the sequences $H_{n}$ and $A_{n}$ uniquely;

(vi) $H_{n-1}=\left(H_{n}\right)_{r}$ and $A_{n-1}=\left(A_{n}\right)_{r}$;

(vii) $\rho_{H_{n}}=\rho_{H}(A)$.

3. Let $n$ be a positive integer or $n=\infty, 0 \leqq b \leqq \infty$, and $x \in H$. We said that the abstract Cauchy problem of order $n$ at the element $x$ on the interval $[0, b]($ or $[0, \infty)$ if $b=\infty)$ for $A$ on $H$ abbreviated by $\operatorname{ACP}(n, x, b, A, H)$, is solvable if there exists an $n$ times continuously differentiable function $y(t)=y(t, x)$ on the interval $[0, b]$ in $H$ with $y(0, x)=x$ and $y^{(k)}(t)=A^{k} y(t)$ for all integers $0 \leqq k<n+1$. By $\operatorname{ACP}^{*}\left(n, x^{*}, b, A^{*}, H^{*}\right)$ is solvable, we mean the same as above except that continuously differentiable is replaced by strongly continuously differentiable. Define

and

$$
X(n, b)=\{x \in H: \operatorname{ACP}(n, x, b, A, H) \text { is solvable }\}
$$

$$
Y(n, b)=\left\{x^{*} \in H^{*}: \operatorname{ACP}^{*}\left(n, x^{*}, B, A^{*}, H^{*}\right) \text { is solvable }\right\} .
$$

Define $X=S(\infty, \infty)$ and $Y=Y(\infty, \infty)$. 
Suppose $X$ is dense in $H$ and $Y$ is $\sigma\left(H^{*}, H\right)$ dense in $H^{*}$. Then $A$ and $A^{*}$ admit unique solutions for the ACP and ACP* respectively, and $T(t)$ is closable in $H$ with domain containing $X$, where $T(t) x=y(t, x)$ for $x \in X$ and $y(t, x)$ the corresponding solution. Define

$$
\begin{array}{ll}
X(0, b)=\{x \in H: x \in D(T(t)) & \text { for all } 0 \leqq t \leqq b \text { and } \\
& T(t) x \text { is continuous in } H\}
\end{array}
$$

and

$$
\begin{aligned}
Y(0, b)=\left\{x^{*} \in H^{*}: x^{*} \in D\left(T(t)^{*}\right)\right. & \text { for all } 0 \leqq t \leqq b \text { and } \\
& \left.T(t)^{*} x^{*} \text { is strongly continuous }\right\}
\end{aligned}
$$

Let $0 \leqq c \leqq \infty$. The function $x^{*} \rightarrow \sup \left\{\left\|A^{* k} T(t)^{*}\right\|: 0 \leqq t \leqq c, 0 \leqq k \leqq n\right\}$ induces a Banach norm on $Y(n, c)$. By the $\sigma\left(H^{*}, H\right)$ denseness of $\bar{Y}$ in $H^{*}, H$ can be naturally identified as a subspace of $Y(n, c)^{*}$. Denote by $H(n, c)$ the closure of $H$ in $Y(n, c)^{*}$ with the subspace topology. By $H(n, \infty)$, we mean the complete, barreled, bornological, locally convex space which is the completion of $H$ with the topology induced by the intersection of all the subspace topologies from $H(k, c)$ for all $c \geqq 0$ and integers $0 \leqq k<n+1$. We summarize some of the properties of $H(n, b)$ in the following.

Theorem 2. Suppose $X$ is dense in $H$ and $Y$ is $\sigma\left(H^{*}, H\right)$ dense in $H^{*}$. Then there exists a family of Banach spaces $H(n, b)$ for $0 \leqq b<\infty$ and integers $0 \leqq n<\infty$, and a family of complete, barreled, bornological 1.c.s. $H(n, \infty)$ for all integers $0 \leqq n \leqq \infty$ such that

(i) $H(0,0)=H$;

(ii) $A$ can be extended as a continuous linear transformation from $H(n, c)$ to $H(n+1, a)$ for all $0 \leqq c \leqq a \leqq \infty$ and integers $0 \leqq n \leqq \infty$;

(iii) $H(n, b)^{*}=Y(n, b)$ for all $0 \leqq b \leqq \infty$ and integers $0 \leqq n \leqq \infty$;

(iv) for each $0 \leqq t \leqq a-c$ and $0 \leqq n<m<\infty, T(t)$ can be extended as $a$ continuous linear transformation from $H(n, c)$ to $H(m, a)$, and $T(t)$ is $m-n-1$ times strongly continuous differentiable;

(v) for each $0 \leqq n \leqq \infty, T(t)$ can be extended as a continuous linear transformation from $\bar{E}_{n}$ to $E_{n}$, and for each $x \in H, T(t) x$ is $n$ times continuously differentiable in $E_{n}$.

4. As an application of the constructions above, we state the following theorems which can be proved by modifying some of the ideas in Fujiwara [11], Chazarain [4] and Beals [2], [3]. We say that a closed operator admits an extended semigroup structure if $X$ is dense in $H$ and $Y$ is $\sigma\left(H^{*}, H\right)$ dense in $H^{*}$. Let $C(A)=\{$ All the continuous operators on $H$ commuting with $A$ \}. 
THEOREM 3. Suppose there exists a real number $w$ such that for all $\lambda$ with $\operatorname{Re} \lambda>w, R(\lambda, A)$ exists, and $\|R(\lambda, A)\| \leqq M(1+|\lambda|)^{k}$ for some $M>0$ and some positive integer $k \geqq 0$. Then

(i) A admits an extended semigroup structure.

(ii) For each integer $n \geqq 0, E_{n}$ can be identified as a subset of $H_{n+k+2}$, and $X(n, \infty)$ contains $H_{-n-k-2}$.

(iii) For each integer $n$, consider $T(t)$ as a continuous linear transformation from $H_{n}$ to $H_{n+k+2}$. Then $T(t)$ is in the closure of $C(A)$ ( $\subseteq$ $\left.L\left(H_{n}, H_{n+k+2}\right)\right)$, and there exists $M_{n}>0$ such that $\|T(t)\|_{H_{n} \rightarrow H_{n+k+2}} \leqq M_{n} e^{r t}$ for all $r>w$ and $0 \leqq t<\infty$.

THEOREM 4. Suppose there exist real numbers $M, \alpha>0, \beta \geqq 1$ and an integer $k \geqq 0$ such that for all $\lambda$ with $\operatorname{Re} \lambda \geqq \alpha \log \beta(1+|\lambda|), R(\lambda, A)$ exists, and $\|R(\lambda, A)\| \leqq M(1+|\lambda|)^{k}$.

(i) $A$ admits an extended semigroup structure;

(ii) for each integer $n \geqq 0$ and $m \geqq 1, H(n, t)$ can be identified as a subset of $H_{k+n+m}$, and $X(n, t)$ contains $H_{-k-n-m}$ for all $0 \leqq t<(n-1) / \alpha$.

THEOREM 5. Suppose there exist constants $\alpha, \beta>0$ and $1>\delta>0$, such that $R(\lambda, A)$ exists for all $\lambda$ with $\operatorname{Re} \lambda \geqq \alpha|\lambda|^{\delta}+\beta$, and for all $\varepsilon>0$ there exists $M, \gamma>0$ such that $\|R(\lambda, A)\| \leqq M \exp \left(\varepsilon \operatorname{Re} \lambda+\gamma|\lambda|^{\delta}\right)$. Then $A$ admits an extended semigroup structure.

\section{REFERENCES}

1. V. Barbu, Differentiable distribution semigroups, Ann. Scuola Norm. Sup. Pisa (3) 23 (1969), 413-429. MR 41 \#2456.

2. R. Beals, On the abstract Cauchy problem, J. Functional Analysis, 10 (1972), 281-299.

3. - Semigroups and abstract Gevrey spaces, J. Functional Analysis 10 (1972), 300-308.

4. J. Chazarain, Problèmes de Cauchy abstraits et applications à quelques problèmes mixtes, J. Functional Analysis 7 (1971), 386-446. MR 43 \#2570.

5. I. Ciorănescu, Sur les semigroupes ultra-distributions, J. Math. Anal. Appl. 41 (1973), 539-544.

6. G. Da Prato and U. Mosco, Semigruppi distribuzioni analitici, Ann. Scuola Norm. Sup. Pisa (3) 19 (1965), 367-396. MR 32 \#2899.

7. G. Da Prato and E. Giusti, Equazioni di Evoluzione in $L^{p}$, Ann. Scuola Norm. Sup. Pisa (3) 21 (1967), 485-505. MR 37 \#5552.

8. N. Dunford and J. T. Schwartz, Linear operators. I: General theory, Pure and Appl. Math., vol. 7, Interscience, New York, 1958. MR 22 \#8302.

9. H. O. Fattorini, A representation theorem for distribution semigroups, J. Functional Analysis 6 (1970), 83-96.

10. C. Foiaş, Remarques sur les semigroupes distributions d'opérateurs normaux, Portugal. Math. 19 (1960), 227-242. MR 26 \#610.

11. D. Fujiwara, A characterization of exponential distribution semigroups, J. Math. Soc. Japan 18 (1966), 267-274. MR 33 \#6420. 
12. E. Hille and R. S. Phillips, Functional analysis and semigroups, Amer. Math. Soc. Colloq. Publ., vol. 31, Amer. Math. Soc., Providence, R.I., 1957. MR 19, 664.

13. H. Komatsu, Semigroups of operators in locally convex spaces, J. Math. Soc. Japan 16 (1964), 230-262. MR 32 \#368.

14. T. Kômura, Semigroups of operators in locally convex spaces, J. Functional Analysis 2 (1968), 258-296. MR 38 \#2634.

15. S. G. Kreĭn, Linear differential equations in Banach space, Nauka, Moscow, 1967; English transl., Transl. Math. Monographs, vol. 29, Amer. Math. Soc., Providence, R.I., 1972. MR 40 \#508.

16. E. Larsson, Generalized distribution semigroups of bounded linear operators, Ann. Scuola Norm. Sup. Pisa (3) 21 (1967), 137-159. MR 38 \#572.

17. J. L. Lions, Les semigroupes distributions, Portugal. Math. 19 (1960), 141-164. MR 26 \#611.

18. I. Miyadera, Semigroups of operators in Fréchet space and applications to partial differential equations, Tôhoku Math. J. (2) 11 (1959), 162-183. MR 21 \#7445.

19. J. Peetre, Sur la théorie des semigroupes distributions, Sem. Leray College de France (1963/1964), 79-98.

20. H. H. Schaefer, Topological vector spaces, Macmillan, New York; CollierMacmillan, London, 1966. MR 33 \#1689.

21. L. Schwartz, Lectures on mixed problems in partial differential equations and the representation of semigroups, Tata Inst. Fund. Res., 1958.

22. M. Sova, Problème de Cauchy pour équations hyperboliques opérationnelles à coefficients constants non-bornés, Ann. Scuola Norm. Sup. Pisa (3) 22 (1968), 67-100. MR 38 \#437.

23. T. Ushijima, Some properties of regular distribution semigroups, Proc. Japan Acad. 45 (1969), 224-227. MR 40 \#7867.

24. $\mathrm{K}$. Yoshinaga, Ultra-distributions and semigroup distributions, Bull. Kyushu Inst. Tech. Math. Natur. Sci. No. 10 (1963), 1-24. MR 28 \#466.

25. K. Yoshida, Functional analysis, Die Grundlehren der math. Wissenschaften, Band 123, Academic Press, New York; Springer-Verlag, Berlin, 1965. MR 31 \#5054.

Department of Mathematics, Duke University, Durham, North Carolina 27706 